$34.1 \mid 2009$

Varia

\title{
L'intemporel incarné : les corps des tourbières entre métaphore et littéralité
}

\section{Valérie Morisson}

\section{Q OpenEdition \\ 12 Journals}

\section{Édition électronique}

URL : http://journals.openedition.org/etudesirlandaises/1222

DOI : 10.4000/etudesirlandaises. 1222

ISSN : 2259-8863

Éditeur

Presses universitaires de Rennes

\section{Édition imprimée}

Date de publication : 30 juin 2009

Pagination : 69-81

ISBN : 978-2-7535-0935-1

ISSN : 0183-973X

Référence électronique

Valérie Morisson, "L'intemporel incarné : les corps des tourbières entre métaphore et littéralité ", Études irlandaises [En ligne], 34.1 | 2009, mis en ligne le 30 juin 2011, consulté le 19 avril 2019. URL http://journals.openedition.org/etudesirlandaises/1222 ; DOI : 10.4000/etudesirlandaises.1222

Ce document a été généré automatiquement le 19 avril 2019.

(c) Presses universitaires de Rennes 


\title{
L'intemporel incarné : les corps des tourbières entre métaphore et littéralité
}

\author{
Valérie Morisson
}

«Et rien ne meurt. De nouvelles choses arrivent; de grands changements surviennent. Mais ce qui était là auparavant ne disparaît pas vraiment ; cela s'enfonce un peu plus et disparaît de la surface. La culture irlandaise est sédimentaire, faite de couches d'expérience et d'émotions superposées les unes aux autres ${ }^{1}$. » L'écho que reçurent les corps exhumés dans des tourbières irlandaises semble donner raison à Fintan O'Toole : Seamus Heaney n'est pas le seul Irlandais dont l'œuvre réfracte ces découvertes archéologiques. Les tourbières ont livré des vestiges majeurs à l'origine d'un ample phénomène culturel. R. C. Turner concluait que « rien ne peut expliquer l'ampleur du phénomène provoqué par les corps des tourbières... Les explications avancées nous apprennent davantage sur l'expliquant que sur le phénomène lui-même ${ }^{2}$ ». Nous lui donnerons raison mais, au-delà des premières ondes de choc qui ont secoué le public après les révélations des archéologues, ne doit-on pas voir dans ces cadavres et leurs évocations plastiques une manifestation mnémotopique éclairant la question irlandaise d'un jour nouveau?

2 Les corps des tourbières constituèrent d'abord un objet d'étude pour les archéologues. En 1965, l'ouvrage de P. V. Glob narrant les découvertes danoises, fit sensation ${ }^{3}$. Les photographies accompagnant le texte étaient si stupéfiantes qu'elles marquèrent les lecteurs et les chercheurs du monde entier, au point que l'auteur s'en étonna lui-même ${ }^{4}$. Les corps extrêmement bien préservés devinrent une source d'inspiration pour plusieurs artistes : parmi les premiers lecteurs de cet ouvrage liminaire figuraient le poète Seamus Heaney et le peintre Louis le Brocquy. En 1969, lorsque parut la traduction anglaise de ce récit archéologique, dix-neuf corps avaient été retrouvés en Irlande. Subséquemment, des expositions firent connaître au grand public ces corps qui font aujourd'hui partie de la culture populaire: les cadavres de l'Age de Fer furent le sujet d'un film d'horreur et plusieurs sites internet y furent consacrés ${ }^{5}$. En 2002-2003, une exposition itinérante intitulée The Mysterious Bog People circula Outre-Atlantique et présenta six corps dont 
deux avait acquis le statut de stars de l'archéologie grâce à la presse quotidienne : celui d'Yde, portant autour du cou la corde intacte qui servit à l'étrangler il y a plus de 2000 ans et celui de Red Franz, momie de 1500 ans fort célèbre en Allemagne pour avoir gardé une crête de cheveux teintés en rouge. L'exposition à grand succès mêlait culture populaire et scientifique : la thématisation du mystère mettait au premier plan le travail de collecte et d'interprétation des archéologues tout en aiguisant la curiosité du public; dans une partie de l'exposition nommée "Bog Science Investigation", les jeunes visiteurs du National History Museum de Los Angeles pouvaient participer à des enquêtes fictives et découvrir les méthodes d'investigations des médecins légistes. Le succès de l'exposition est dû autant aux choix muséographiques, privilégiant la participation du visiteur, qu'à l'attrait plus morbide pour les cadavres en «chair et en os" du mésolithique. L'archéologue danois Winjand van der Sanden s'émut de voir les corps reproduits sur les produits dérivés de l'exposition et jugea la transformation de cadavres d'ancêtres glorieux en objets de consommation peu conforme à l'éthique muséographique ${ }^{6}$.

Depuis le début du dix-neuvième siècle, les tourbières irlandaises ont livré 89 corps. Le premier document relatif à ces découvertes, rédigé par Lady Moira en $1781^{7}$, provient du comté de Down. Seule une minorité des cadavres trouvés en Irlande remonte à la préhistoire. Les premières découvertes d'objets de l'Age du Bronze (1880-700 av. J.-C.) datent des années 1830 ; la plupart des corps déterrés alors étaient rejetés et échappaient à l'analyse scientifique. L'homme de Gallagh (470-120 av. J.-C.) fut retrouvé en 1821 non loin de Castleblakeney (Co. Galway) et transféré au National Museum en 1829. Ce n'est qu'en 1978, après la découverte à Meenybradden (Co. Donegal) d'un corps daté de 1570 dont la partie supérieure était encore recouverte de peau que des recherches archéologiques furent menées. Faisant suite aux travaux de Glob et Wijnand van der Sanden au Danemark ${ }^{8}$, Barry Raftery, spécialiste irlandais de l'Age de Fer et de l'art celte, publia Trackways Through Time ${ }^{9}$. En Irlande, l'intérêt pour les corps momifiés des tourbières fut ravivé en 2003 après l'exhumation accidentelle de deux corps morcelés, retrouvés à vingt-cinq kilomètres d'écart sur des exploitations de tourbe : à Croghan, celui d'un homme ayant vécu aux environs de 362-175 av. JC, à Clonycavan, plus fragmentaire, celui d'un homme mort il y a 2300 ans. La nouvelle des découvertes fut rapportée non seulement dans des revues spécialisées mais également par The Mirror ou l' Irish Times. L'émission Time Watch de la BBC, diffusée en janvier 2006, revint en détail sur les conclusions des archéologues et vulgarisa les résultats de l'enquête dirigée par Isabella Mulhall, coordonatrice du Bog Bodies Project. Trois mois plus tard, l'exposition du National Museum de Dublin, Kingship and Sacrifice - an Exhibition of Bog Bodies and Related Finds, présenta au public ces deux découvertes et appuya la thèse selon laquelle ces sacrifices, pratiqués près des limites territoriales, étaient liés à l'exercice du pouvoir royal. Melanie Giles, chercheuse à l'université de Manchester remarqua à propos des deux corps découverts en 2003: "Perhaps, unsurprisingly, they have captured the imagination of poets, writers and film-makers, who have responded evocatively to the drama and pathos of such encounters ${ }^{10}$."

4 Les Bog Poems de Seamus Heaney ont aiguisé l'intérêt de plusieurs chercheurs ${ }^{11}$. Néanmoins, peu de cas est fait de la référence à ces corps dans les arts visuels. Or, le nombre d'œuvres directement inspirées de ces découvertes atteste et explique l'imaginaire très spécifique que firent naître ces étranges revenants. L'évocation des corps dans l'art contribua à transformer une découverte archéologique en fait culturel en prise avec le présent. 


\title{
Une faille chronologique
}

5 En raison de l'état de conservation exceptionnel des corps et des visages, les découvertes provoquèrent un inhabituel écrasement temporel. L'acidité des tourbières, ainsi que l'absence d'oxygène dans le sous-sol permit la conservation des tissus organiques (peau, cheveux, ongles, organes) et de matériaux tels que la laine ou le coton. Les photographies de corps publiées par Glob donnent à voir chaque ride, des poils de barbe et des sourcils ; l'expression des visages est parfois saisissante. Les corps se distinguent donc très nettement des gisants ou des masques funéraires. L'une des photographies est ainsi légendée : "The dead and the sleeping, how they resemble one another ${ }^{12}$ ", légende qui ne peut que rappeler les vers de Seamus Heaney : «Who will say 'corpse'/to his vivid cast?/Who will say 'body'/to his opaque repose ${ }^{13}$ ?"

6 Au milieu des années 1960, P. V. Glob avait noté des erreurs quant à la datation des corps : " As they worked, they suddenly saw in the peat-layer a face so fresh that they could only suppose they had stumbled on a recent murder ${ }^{14}$. » En Irlande, la confusion fut alimentée par le fait que les tourbières servirent à cacher les corps de victimes des Troubles dans les années 1960-1970. Le corps parfaitement conservé de l'homme de Croghan fit d'abord l'objet d'une enquête policière car on pensait qu'il s'agissait du corps d'une victime récente ${ }^{15}$. Rolly Reed, l'un des conservateurs chargés de l'enquête, revint sur ses émotions lors d'une interview :

\begin{abstract}
I had visions of these enormous arms coming round the back of my neck...I was getting flashbacks for a fortnight... I was having nightmares. What hit me hardest, I think, was the fingerprints perfect fingerprints - the same as a guy's from today. He could have been anybody off the streets of Dublin... it was like touching your own skin ${ }^{16}$.
\end{abstract}

7 L'écrasement temporel est accentué par les reconstitutions scientifiques, la connaissance des individus meurtris et la présentation des corps dans le musée. Le buste de l'homme de Clonycavan a été remodelé par les artistes du département de criminologie de l'Université de Dundee. On connait aujourd'hui jusqu'au menu du dernier repas de l'homme de Croghan. Devant ces corps exposés dans un musée, le visiteur est confronté au temps historique de la mise à mort mais également au temps de l'exhumation et de la redécouverte car les corps sont présentés exactement comme ils ont été trouvés. Plusieurs éléments de la mise en scène muséographique créent une intimité déconcertante entre le spectateur et le mort et nous invitent à imaginer la victime avant son exécution. Glob conclut d'ailleurs sa description de l'homme de Tollund ainsi : «Dark in hue, the head is still full of life and more beautiful than the best portraits by the world's greatest artists, since it is the man himself we see ${ }^{17}$. "

8 Les corps des tourbières incarnent et matérialisent un passé qui se confond étrangement avec le présent. S'inspirant du chronotope de Bakhtine ${ }^{18}$, Anthony Purdy conceptualisa la notion de mnémotope, que les corps des tourbières incarnent :

Since the primary function of the archaeological artifact as chronotope is to materialize a past in the present, to serve as a vehicle for personal and cultural memory, I will refer to it as a 
mnemotope, a term that should be fairly transparent but that I will define provisionally as a chronotopic motif manifesting the presence of the past, the conscious or unconscious memory traces of a more or less distant period in the life of a culture or, metaphorically, an individual. of course, the mnemotope might come in many guises and be inflected by attitudinal values ranging from nostalgia and melancholy through desire, obsession and remembrance to horror and denial. [...] To an extraordinary degree, bog bodies allow us to see time ${ }^{19}$.

9 Karine Sanders, de l'université de Berkeley, note pareillement une fusion du passé et du présent : "Whether you're looking at it as a scientist or a poet, it's very easy to imagine the bog bodies as a direct portal into the past. The bodies make history seem very close. It's as if we're looking right into the face of our ancestors ${ }^{20}$. " L'impression est proche de celle qu'exprime Turner: "The sense of wonder [bog bodies] conjure up is combined with the feeling that they have in some way cheated death, to live again ${ }^{21}$. " Plus que tout autre vestige archéologique, le corps semble à même de faire resurgir le passé dans le présent. L'écrivain Eugène Nshimiyimana, parlant de cadavres meurtris, affirme que "le corps se situe nécessairement dans une double dimension, temporelle et atemporelle, qui l'associe à la mémoire dans son rapport au passé et au futur ${ }^{22}$ ». Le temps est matérialisé sur le corps par des traces de coups, des cicatrices, des attitudes, il devient un espace mémoriel, au même titre que les lieux de mémoire évoqués par Pierre Nora ${ }^{23}$. Cependant, contrairement à l'acte commémoratif qui implique une volonté, voire un geste, d'ordre politique, le mnémotope surgit de manière involontaire, tel le corps d'une victime torturée refaisant surface au milieu d'un paysage pastoral.

C'est en tant que mnémotopes que les corps des tourbières sont représentés par l'artiste Barrie Cooke (né en 1931), intéressé par les écosystèmes et les biotopes autant que par l'animisme qu'il a découvert par ses voyages (Nouvelle-Zélande, Bornéo, Java) ou par le taoïsme ${ }^{24}$. Dans plusieurs œuvres (Bog, 1970, Turf Box, 1974 et Bog Box, 1975), la tourbe n'est pas un symbole de l'identité nationale mais un milieu géologique et biologique particulier qu'il peint après l'avoir observé avec l'œil d'un biologiste. La série de toiles intitulées Bog Figures, I - VI, datées de 1973, montre que Cooke fut influencé par le livre de P. V. Glob et fasciné par les conditions de préservation des tissus : « His response to The Bog People was in large measure derived from the fact that it described things which dovetailed with his own concerns and intuitions ${ }^{25}$. On observe une similarité frappante entre les toiles et les photographies de Glob - le peintre s'efforçant de suggérer l'ensevelissement du corps dans un milieu géologique qui l'absorbe. Cooke produisit plusieurs dessins destinés à illustrer les Bog Poems de Seamus Heaney. Le regard de Cooke sur les corps est presque scientifique: les cadavres ou corps endormis qu'il représente sont tels des fossiles, parfaitement enchâssés dans le sol spongieux. Parfois, une simple accumulation de matière plus qu'une variation chromatique signale la présence du corps dans le sol. Les corps, nus, sont peints comme des organismes faits de tissus et d'articulations. L'épaisseur de la pâte imite les fibres musculaires et les ligaments, et dessine des linéaments sinueux semblables à des racines ou des rhizomes si bien que le corps semble englouti par la terre :

There is an extraordinary intimacy between human and peat. Preserved through a kind of tanning process, the bog peoples' skin 
darkens to leathery beauty, towards the black, moist turf. [...] Bog figures and bone boxes alike suggest something ancient and dormant revivified, and they could be read as metaphors of primitive consciousness, more directly in touch with a world of natural forces ${ }^{26}$

西 Tollund Man, the Winderby Girl, and others sacrificed in an Iron Age bog that had preserved them perfectly to the inclination of an eyelid. Yes, I'm afraid that 'terrible' aspect may sometimes be reflected in my work ${ }^{27}$. " Ses séries de têtes ou portraits déformés doivent autant aux têtes polynésiennes qu'il découvre au musée de l'Homme à Paris en 1964, qu'aux têtes qu'il voit l'année suivante sur le site celte ligurien d'Entremont en Provence ou aux photographies des corps des tourbières publiées par Glob ${ }^{28}$. Le travail de le Brocquy illustre une transcendance, en ce qu'il a la vertu de présentifier l'absent : " These portraits see death as a part of life, within the flux of the continuous present ${ }^{29}$. " Les visages démantelés, décomposés, ne sont pas tant une évocation de la mort qu'une tentative d'accéder à l'esprit logé dans le crâne, à la spiritualité piégée dans l'enveloppe matérielle de la tête. Louis le Brocquy travaille là où le visible se fissure pour révéler l'esprit : « For our Celtic ancestors, I imagine, the head was a box which holds the spirit prisoner but which at the same time manifests this spirit. The head is simultaneously a mask which hides the mind and a revelation, an incarnation of mind. [...] I try to scuffle the surface of the paper or the canvas, to penetrate the surface rather like an archaeologist in order to see what may emerge ${ }^{30}$. » À propos de la main de Louis le Brocquy, Seamus Heaney évoque aussi l'homme de Tollund, dont le corps l'a pareillement marqué :

13 L'idée de permanence, d'absence de temporalité est évoquée par l'artiste lui-même. Les visages fragmentés sont les métaphores d'une temporalité qui se dissout. Le mélange de passé mythologique et de modernité avant-gardiste dans l'éclatement de la figure aboutit à une déconstruction de la linéarité chronologique.

\section{De la fracture temporelle à la béance visuelle}

14 Les bog people font resurgir une violence jusque-là enfouie et qui, lorsqu'elle réapparaît à la surface, évoque soit une éternelle béance de l'enveloppe corporelle soit l'inscription des meurtres contemporains dans une mythologie nationale.

15 Comme l'ont montré les analyses des médecins légistes et des archéologues, les personnes dont le corps avait été exhumé avaient été victimes de violences multiples ${ }^{32}$. L'homme de Gallagh (trouvé à Castleblakeney et conservé au National Museum de Dublin) avait été étranglé et maintenu dans le sol par deux piquets de bois. D'autres corps retrouvés en Irlande ont été éviscérés et démembrés. C'est le cas de l'homme de Croghan, dont la tête 
et certains membres étaient manquants. Sa poitrine était lacérée, il avait été attaché grâce à deux trous percés dans son bras et blessé à plusieurs reprises à l'estomac. Ces perforations corporelles, qui sont de précieux indices pour les archéologues, sont autant de brèches dans lesquelles s'engouffre un imaginaire de la violence attisé par le mystère des crimes. Les corps sont identifiés comme étant ceux de victimes tantôt de sacrifices destinés à célébrer des victoires militaires, à encourager la fertilité ou à aider à une guérison (Glob) ; tantôt de châtiments infligés par la communauté entière après une transgression des codes sociétaux ${ }^{33}$. Parfois encore, les corps sont présentés comme ceux d'individus victimes d'un bannissement hors du périmètre de la paroisse ${ }^{34}$ ou de rituels associés au pouvoir royal (Ned Kelly), ou encore d'actes divinatoires, ce qui expliquerait les éviscérations. Les corps seraient donc ceux de boucs émissaires choisis pour incarner la culpabilité de leur communauté ou ceux de captifs otages de guerres claniques ou tribales. Quelle que soit l'interprétation, les corps apportent la preuve de pratiques culturelles qui tolèrent la cruauté comme moyen d'améliorer le sort de la communauté. Les photographies des corps publiées par les archéologues et les journaux ne masquèrent guère la brutalité, et l'on peut avancer que c'est cette cruauté brute qui frappa sans doute le plus les artistes.

Hughie O'Donoghue, né à Manchester en 1953 d'un père soldat, réalise un travail sériel sur le corps contorsionné, déformé et maculé par des cicatrices, des marques, des taches. Ses toiles sombres, qui refusent de montrer des visages ou un lieu, « s'efforcent de donner une impression de corporéité, une sensation d'étouffement, d'intoxication, de nausée, de douleur, l'impression que membres et muscles sont en mouvement, que l'on touche la peau, le toucher, la succion, les morsures, la conscience d'organes internes et des tissus ${ }^{35}$ ». Fasciné par Titien et Le Greco, O'Donoghue l'est aussi par le sous-sol irlandais et ses marécages. La série de toiles intitulées Sleeper (commencée dès 1984) fut influencée par la découverte de l'homme de Tollund. L'artiste explique qu'enfant, on lui racontait des histoires sur ces hommes au passé obscur. Les toiles semi-abstraites aux couleurs sombres de Tomb et Within Earth (1980-1990) illustrent une angoisse de l'engloutissement, de l'enterrement. Dans Blue Crucifixion (1993-2003), toile de plus de huit mètres de long, le fond bleu maculé de noir laisse entrevoir une vague silhouette humaine qui ressemble à une empreinte cireuse ou à une momie :

A theme, or image, which has haunted them [his paintings] for several years is that of a human figure, often implicit rather than defined, which at times obviously relates to the crucifixion, at other times to the "bog figures" which have inspired Seamus Heaney. [...] Here perhaps, we have an image of what lies buried in our unconscious, and also an expression of the regenerative powers of nature and of the complex, many-layered myth of the Resurrection ${ }^{36}$.

17 Si la position des corps rappelle les squelettes des musées archéologiques, la stylisation des cicatrices et l'utilisation d'une palette presque monochrome pour accentuer l'intensité du fond invitent à une lecture littérale, aphoristique plus que métaphorique. La série de toiles qu'Hughie O'Donoghue réalisa sur la Passion du Christ dévoile des corps noueux et recroquevillés qui doivent autant aux corps des tourbières qu'aux torsions des figures de Rembrandt ${ }^{37}$. Des tons sombres et rougeoyants baignent ces scènes intemporelles qui évoquent la fragilité primordiale du corps : « The work of art becomes the 
medium that carries the predilection of the artist for the present time, a time in which the past comes back to life, although in a very peculiar and precarious way ${ }^{38}$."

o'Donoghue fait émerger de la toile l'archétype du corps blessé, agonisant, qui hante l'art et la littérature. La qualité centripète des aplats au couteau, l'éclatement des touches et la taille des toiles matérialisent la souffrance du corps. David Slattery voit dans la blessure la présence violente du sacré et du mythe qui entre en nous par l'acte de l'Autre. La faille dans le corps serait le locus privilégié de notre rencontre, fut-elle violente, avec le sacré, le langage et le social. Le corps doit se penser comme un lieu culturellement marqué : « In the body distended and distorted there is revealed something true about the interaction of individuals and the world concerning their own fatedness [sic], their individual destiny, and their evolving character ${ }^{39}$." Les corps meurtris des tourbières et les êtres scarifiés et contorsionnés de O'Donoghue ont ceci de commun qu'ils constituent des images culturelles, sièges de mythologies individuelles et collectives. La blessure rend visible la vulnérabilité des hommes et aiguise notre conscience du danger, de l'impermanence, de l'incessante confrontation au monde et à autrui. Les coups, les écorchures, les plaies et autres atteintes à l'intégrité corporelle tracent aussi des parcours d'initiation et construisent notre identité. La cicatrice inscrit le corps dans le temps et la narrativité : "[...] signing or marking the body signifies its passage into writing, its becoming a literary body, and generally also a narrative body, in that the inscription of the sign depends on and produces a story. The signing of the body is an allegory of the body become a subject for literary writing - a body entered in writing ${ }^{40}$. »

\section{De la métaphore archéologique au symbole politique}

La pratique de la vidéo resitue le corps dans une trame narrative qui se prête particulièrement au jeu sur la temporalité, la focalisation et l'identité. Tout comme Seamus Heaney, Pauline Cummins, vidéaste et photographe irlandaise, a évoqué les victimes sacrificielles de l'Age du Bronze en relation avec le conflit nord-irlandais au début des années 1980 : «I tried to look further back into our history, for ancient symbols, and past rituals, in an attempt to rise above and view the present, as a tiny part of an ongoing cycle. I became interested in bodies preserved in the bogs, some of them two thousand years old ${ }^{41}$." Pauline Cummins commença alors à réaliser des masques d'argile émiettée en raku, évoquant le passage du temps et la décrépitude. Ces visages étaient immortalisés dans l'action, leurs expressions saisies sur le vif, comme ceux de victimes d'attaques imprévues, d'où leur lien avec le terrorisme en Irlande : «My clay figures speak of the past, and of the present, of life remembered -life dismembered ${ }^{42}$." Dans Unearthed (Fig. 1), installation de 1988 centrée sur le thème des bog people, l'artiste proposa de réfléchir aux résurgences du passé dans la vie politique actuelle. Dans une vidéo, l'artiste, vêtue de noir, interroge le public en adoptant un accent irlandais prononcé : «Irish? Me? I’m not Irish... » - puis, retrouvant un accent anglais, évoque la nationalité anglaise de sa mère, puis le Dimanche Sanglant de 1972, avant d'entonner un chant de lamentation traditionnel, le keening ${ }^{43}$. À l'écran, le spectateur voit des visages qui se transforment en masques d'argile ou de raku disposés dans un paysage naturel verdoyant, ainsi que des photos personnelles de l'artiste. Les craquelures de l'émail ressemblent à des cicatrices sur des visages déformés : "With their eye slits, twisted mouth holes and missing noses, the masks are at once bog people and the bandaged, Northern dead who won't stay buried. They're waiting for a cease-fire ${ }^{44}$. $)$ Unearthed présente des versions contradictoires du conflit. 
Cummins entend montrer que personne ne détient la vérité même si chacun doit être responsable de ses actes ${ }^{45}$. La pratique de l'installation, rompant avec l'unité de l'espace de représentation et permettant des juxtapositions non congruentes, est particulièrement propice à instiller le doute dans l'esprit du spectateur qui doit reconstruire le sens de ce qui se présente comme hybride et épars.

Figure 1 : Pauline Cummins, Raku Mask, Unearthed (1988) - Performance et installation.

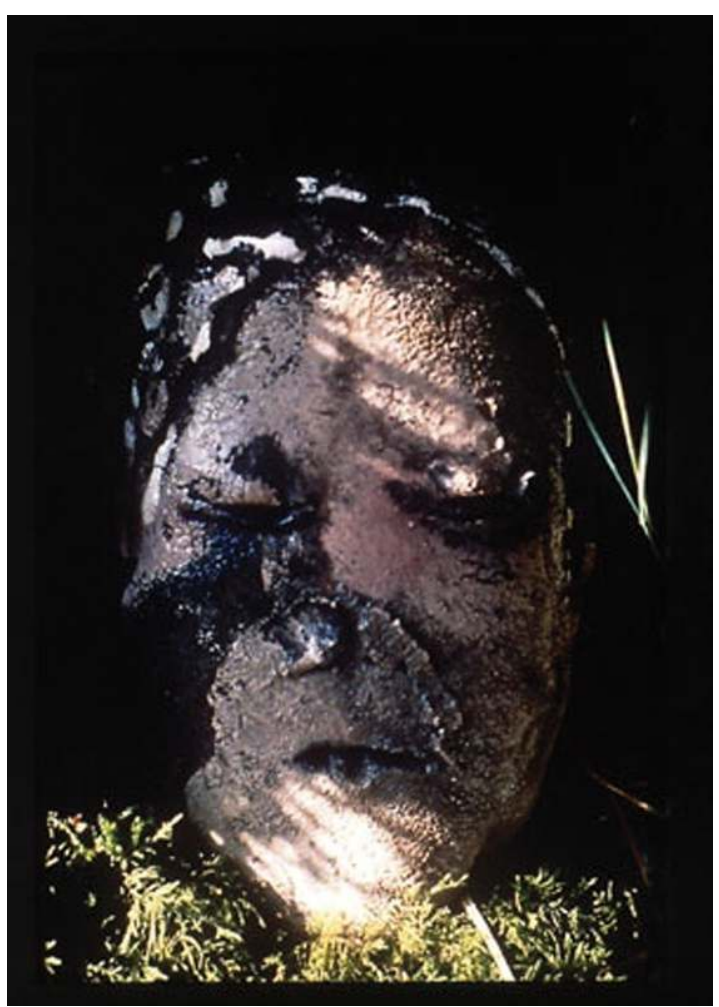

P. Cummins précise dans un courriel adressé à l'auteur en février 2009 : « The work Unearthed was a multimedia performance commissioned by Projects UK in 1988, and later became a photographic and video installation for the exhibition, Inheritance And Transformation, The Irish Museum of Modern Art, 1991. » (Reproduction avec l'aimable autorisation de l'artiste)

20 Autre vidéaste, Nigel Rolfe travaille inlassablement sur la mémoire ainsi que sur la construction de l'identité et de la culture nationales. Marqué par le positionnement politico-social de Joseph Beuys, Rolfe pratique de manière délibérée un art politique. Il s'intéresse aux ruptures plus qu'aux équilibres et s'efforce de poser des questions, "d'ouvrir des blessures ${ }^{46}$ ». L'une de ses expositions inclut le texte suivant : « Expose what is underneath/suggest other possibilities/examine and question/heart and soul/dig deep, unearth, discover/history, memory, imagination ${ }^{47}$. » The Edge of Europe (1988) est une photographie de performance le montrant gisant nu et immobile, vulnérable, dans un marais de tourbe du comté de Sligo : c'est là une image de naissance et de mort, de communion avec le sol qui trouve un écho dans la ruralité si longtemps associée à l'identité irlandaise. L'action faisait référence aux corps des tourbières : " [...] often I have turned to archaeology and the landscape as a source for my work ${ }^{48}$." Rolfe interroge ici la primitivité de l'Irlande, primitivité qui est devenue, au fil de l'histoire nationaliste de l'île, une construction culturelle centrale dans l'identité irlandaise, une légende étayée par l'archéologie.

21 Ni l'archéologie ni le folklore ne sont exempts de biais politiques. Bruce Trigger a mis en évidence des schémas de récits archéologiques communs liés à des contextes politiques 
particuliers ${ }^{49}$. Ainsi, les corps des tourbières sont des accroches pour des mythologies individuelles ou collectives mais aussi des preuves de l'étendue de la culture celte. Or, cet âge d'or celte servit à légitimer des revendications politiques :

Images from the past play conspicuous and powerful roles in the present. [...] Archaeological finds become battle-banners of modern ethnic groups and nations; how the dubious evidence of ancient ethnic migrations and diffusions can be used to legitimize modern territorial expansion and ethnic cleansing; how pattern of archaeological funding and scholarly interest can place interest on certain politically useful sites and certain classes of evidence; and how archaeological interpretation can often both reflect and reinforce the centralizing policies of emerging nation-states ${ }^{50}$.

Les artistes contemporains qui, comme Nigel Rolfe, optent pour une attitude postnationaliste remettent en cause le nationalisme culturel et son influence sur l'iconographie nationale. Ils déconstruisent la culture nationale, interrogent la mise en images, la patrimonialisation et les pratiques mémorielles dans leur rapport à l'élaboration de l'identité irlandaise. En cela, Rolfe est représentatif d'une génération d'artistes se préoccupant des biais politiques, conscients ou inconscients, véhiculés par la culture.

La tranchée initialement tracée dans le champ de tourbe a révélé aux contemporains un passé oublié et, ce faisant, instauré une brèche dans la temporalité. Que le corps déterré soit métaphore ou incarnation littérale, il bouleverse le rapport de l'être au passé ainsi que sa conscience de l'histoire et de son identité. Dans plusieurs performances filmées ou photographiées, Nigel Rolfe, artiste-chaman, s'asperge de farine ou de poussière transformant ainsi son corps en objet étrange. Dust Breeding (2008) ${ }^{51}$ est une vidéo montée en boucle qui provoque un trouble de la temporalité propre au rituel prophylactique. Le visage de l'artiste, couché au sol et filmé en gros plan, est déformé par l'application de la poussière qui se craquelle et se fissure (Fig. 2 et 3). Les œuvres visuelles s'inspirant des corps des tourbières puisent leur force dans la littéralité de la présentation du corps. Alors que les textes de Glob et de Heaney tracent les contours des corps et ressuscitent les individus dont ils font les portraits, les œuvres visuelles nient l'intégrité du corps et dissolvent le sujet dans la matière. La condensation des signes visuels permet d'accéder à des topoï universels. L'enfouissement du visage de Rolfe rappelle l'ensevelissement des corps des tourbières mais aussi le travestissement par le masque et la catharsis qu'il permet. Préférant le primordial au primitif, Rolfe poursuit ainsi une réflexion transculturelle sur l'Autre insaisissable qui se loge au cœur d'un moi familier, sur la barbarie qui resurgit soudain dans la civilisation. Son travail illustre les propos de Mélanie Giles : «Part of the important process of forgetting is the act of remembering ${ }^{52}$. » 
Figures 2 et 3 : Nigel Rolfe, Dust Breeding (2008)
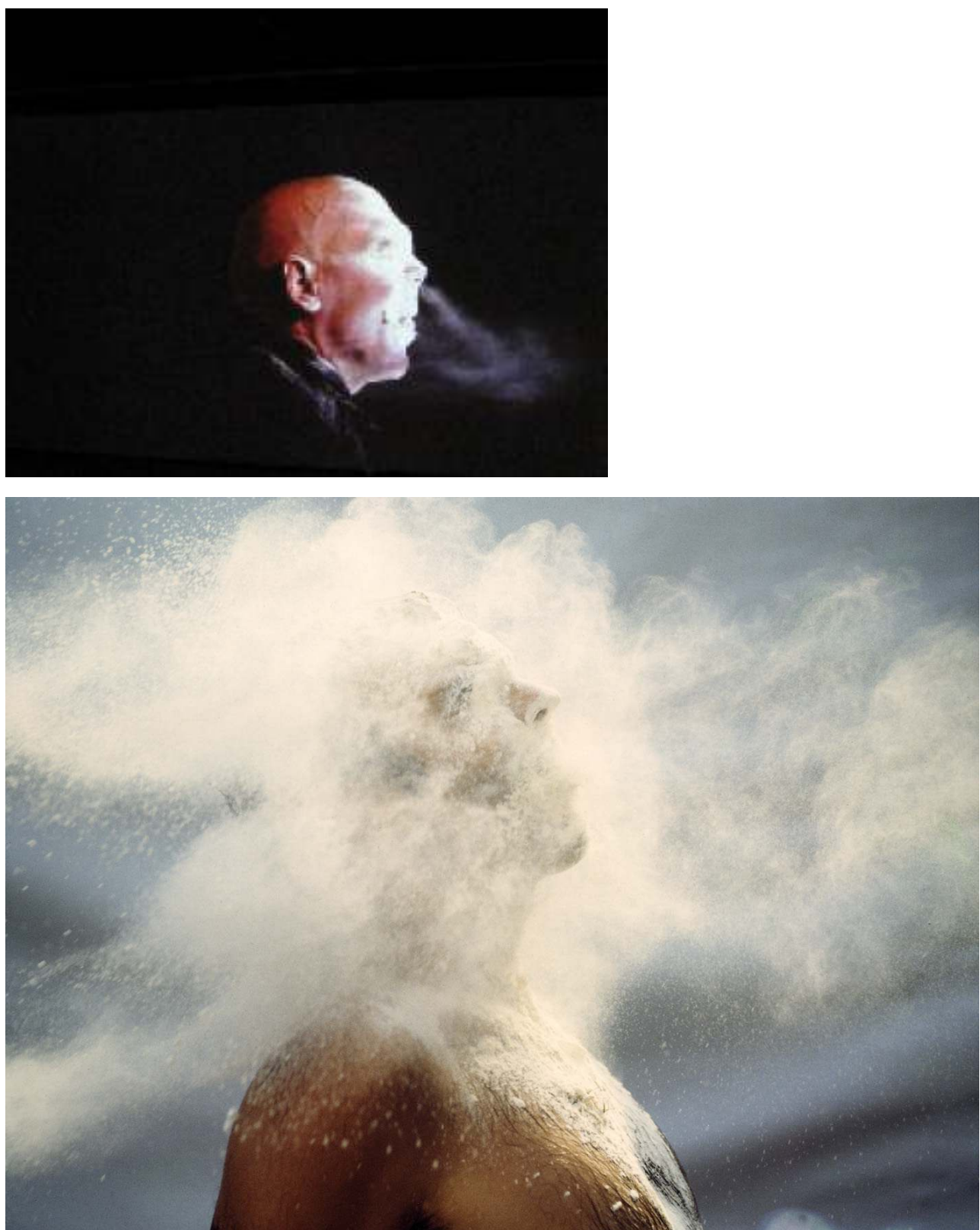

Photographies couleur, 36 × 36in. Droits de reproduction concédés par la Green on Red Gallery, Dublin. Remerciements à Mary Conlon.

\section{NOTES}

1. Fintan O'Toole, "Ireland ", Irish Art Now. From the Poetic to the Political, Boston, Merrell Holberton-McMullen Museum of Art, 1999, p. 23 (traduction de l'auteur). 
2. R. C. Turner, «The Lindow Man Phenomenon: Ancient and Modern», Bog Bodies: New Discoveries and New Perspectives, Londres, British Museum Press, 1995 (traduction de l'auteur).

3. L'ouvrage faisait suite à la découverte, dans des tourbières danoises, de plusieurs corps datant de l'Age de Fer (700-52 av. JC). Le plus vieux était celui de la femme de Koelberg, qui aurait vécu il y a 5500 ans et dont le crâne est aujourd'hui conservé au Fyns Stifsmuseum d'Odense au Danemark (voir Paul Bahn, «Bodies of the Bogs », Archeology, vol. 50, n 4, juillet, 1997, p. 62-67).

4. Glob, The Bog People, Iron-Age Man Preserved, Londres, Faber and Faber, traduit du danois en 1969, p. 15-16.

5. Bog Bodies, le film d'horreur irlandais réalisé par Brendan Foley, fut commercialisé en 2008 (Voir http://www.bogbodiesthemovie.com). Voir également $h$ ttp://mummytombs.com et http://www.tollundman.dk/andre-moselig.asp pour les sites populaires de vulgarisation.

6. Voir Winjnand Van Der Sanden, « Mummies, Mugs and Museum Shops", Archeological Institute of America, 30 août 2005, www.archeology.org/online/features/bog/exhibit.html et Bergen, C., M. J. L. Th. Niekus and V. T. van Vilsteren (dir.), The Mysterious Bog People. Zwolle, Netherlands: Waanders Publishers; Hanover, Germany: Niedersächsisches Landesmuseum Hannover ; Gatineau, Quebec : Canadian Museum of Civilization ; Calgary, Alberta : Glenbow Museum, 2002.

7. Voir P. V. Glob, op. cit., p. 69.

8. Van der Sanden, Wijnand, Through Nature to Eternity. The Bog Bodies of Northwest Europe, Amsterdam, Batavian Lion International, 1996 et « Mummies, Mugs, and Museum Shops", Archeological Institute of America, 30 août 2005, voir note 6.

9. B. Raftery, Trackways Through Time: Archaeological Investigations on Irish Bog Roads, 1985-1989, Rush, Co. Dublin, Headline Publishing, 1990.

10. Giles, Melanie, «Bog Bodies: Representing the Dead», contribution à la conférence intitulée "Respect for Ancient British Human Remains: Philosophy and Practice», 17 novembre 2006, Manchester Museum, http://www.museum.manchester.ac.uk/medialibrary Ldocuments/respect/bog_bodies_representing_the_dead.pdf/.

11. Heaney Seamus, Bog Poems, Londres, Rainbow Press, 1975 ; Door into the Dark, New York, Oxford University Press, 1969 ; Preoccupations, Selected Prose 1968-1978, Londres, Faber \& Faber, 1980, p. 57-58. Voir également David Annwn, Inhabited Voices: Myth and History in the Poetry of Georgy Hill, Seamus Heaney and George Mackay Brown, Frome, Somerset, Bran's Head Books, 1984 ; Anthony Bailey, «A Gift for Being in Touch: Seamus Heaney builds houses of truth", Quest, n², janvier-février 1978; Dianne, Meredith, "Landscape or Mindscape? Seamus Heaney's Bogs", in Irish Geographical Society, vol. 32.2, 1999, p. 127-133 ; Michael Parker, "Gleanings, Leavings : Irish and American Influences on Seamus Heaney's Wintering Out (1972)", in New Hibernia Review, vol.2 : 3, automne 1998.

12. Glob, P. V., op. cit., p. 29.

13. Heaney, Seamus, «The Grauballe Man », in North, Londres, Faber \& Faber, 1975.

14. Glob, P. V., op. cit., p. 18.

15. Voir Claire Raymond, « Murdered 2,500 years ago », The Mirror, 7 janvier 2006.

16. In Grice, E., «A Chilling Tale of Ritual Murder», Daily Telegraph, 7 janvier 2006, p. 19-21, article cité par Giles, M., op. cit., p. 8.

17. Ibid., p. 36. 
18. M. M. Bakhtine, Esthétique et théorie du roman, traduit du russe, Paris, Gallimard, collection «Bibliothèque des idées », 1978.

19. Purdy, Anthony, «The Bog Body as Mnemotope : Nationalist Archaeologies in Heaney and Tournier », Style, printemps 2002.

20. Dayton, Todd, «Tales from the Bog ", Illumination, Berkeley University, 2002, http:// illuminations.berkeley.edu/archives/2002/article.

21. Turner, Richard et Scaife, Robert, Bodies : New Discoveries and New Perspectives, Londres, British Museum Press, 1995, p. 32.

22. Eugène Nshimiyimana, «Les corps mythiques de Sony Labou Tansi », Études Françaises, $41: 2,2005$, p. 95.

23. Voir Pierre Nora (dir.), Les lieux de mémoire, Paris, Gallimard (Quarto), 3 tomes, 1997.

24. [Seamus Heaney, Aidan Dunne et Niall McMonagle], Barrie Cooke, Kinsale, Gandon Edition, coll. Profile, 1998, p. 12.

25. Ibid.

26. Propos de l'artiste, cités dans Dunne, Aidan, Barrie Cooke, Douglas Hyde Gallery, ACNI, 1986, p. 75-76.

27. Morgan, George, "An Interview with Louis le Brocquy by George Morgan », in Le Brocquy, P. (dir.), Louis le Brocquy, The Head Image, Kinsale, Gandon Editions, 1996, p. 17.

28. Ibid.

29. Montague, John, A Louis le Brocquy, Gimpel Fils Gallery, Londres, 1974, p. 3.

30. Le Brocquy, Louis, Images of W. B. Yeats, James Joyce, Federico Garcia Lorca, Picasso, Samuel Beckett, Francis Bacon, 1975-1987, p. 14.

31. Carpenter, Andrew (dir.), Eight Irish Writers, Eight Poems in a Portfolio of Collotype Lithographs, Dublin, 1981, non paginé.

32. Voir Don Brothwell, The Bog Man and the Archaeology of People, Cambridge, Massachusetts, Harvard University Press, 1987 ; Kiner, A. «Momies de l'âge du fer: le peuple des tourbières ", Sciences et avenir, $n^{\circ} 607$, septembre 1997, p. 90-96; Menon, Shanti. "The People of the Bog ", in Discover, août 1997, p. 60-67, 87 ; Mohen, J. P. «Les hommes des tourbières ", in Dossiers d'archéologie, $n^{\circ} 259$, décembre 2000, p. 10-15; Owen, James, "Murdered 'Bog Men' Found With Hair Gel, Manicured Nails ", in National Geographic News, January 17, 2006 ; Pringle, Heather Anne, The Mummy Congress : Science, Obsession, and the Everlasting Dead, New York, Hyperion, 2001.

33. Miranda Green, Dying for the Gods : Human Sacrifice in Iron Age and Roman Europe, Stroud, Tempus, 2001, p. 118.

34. Tim Taylor, The Buried Soul: How Humans Invented Death, Beacon Press, 2004 et Fischer, John Hayes (réal.), The Perfect Corpse, DVD distribué par NOVA, 2006.

35. O'Donoghue, Hughie, 13 Drawings from the Human Body, Jill George Gallery, Londres, 1993, non paginé. (traduction de l'auteur).

36. Fallon, Brian (introduction), A Line of Retreat, Hughie O’Donoghue, Purdy / Hicks, Londres, 1997, p. 7.

37. O’Donoghue, Hughie, Paintings and Drawings 1983-86, Fabian Carlsson Gallery, Londres, 1986, non paginé.

38. Oliva, Achille Bonito, Hughie O’Donoghue, Opere 1986-87, Galleria Carini Firenze, 1987, non paginé. 
39. Slattery, Denis Patrick, The Wounded Body, Remembering the Markings of Flesh, New York, State University of New York Press, 2000, p. 7-11

40. Brooks, Peter, Body Work: Objects of Desire in Modern Narrative, Cambridge, Harvard University Press, 1993, p. 3.

41. Cummins, Pauline, "A Series of Changes », Performance Magazine, 54, juin-juillet 1988, p. 22.

42. Idem.

43. Voir le script de la performance dans Cummins, Pauline, «Unearthed, Performance script; n.paradoxa, vol.5, p. 71-73.

44. Higgins, Judith, « Art From the Edge », in Art in America, décembre 1995, p. 37-40.

45. Deepwell, Kathy, Dialogues, Women Artists from Ireland, Tauris and Company, 2004, p. 154.

46. Nigel Rolfe, Vidéos 1983-1996, interview avec Declan McGonagle, Musée d'Art Moderne de la ville de Paris, 1996 (en français dans le texte).

47. Rolfe Nigel, Resonator, Douglas Hyde Gallery, Dublin, 1992.

48. Rolfe Nigel, Vidéos 1983-1996, op. cit., p. 12.

49. Trigger, Bruce G., " Alternative Archaeologies. Nationalist, Colonialist, Imperialist », in Robert Preucel et Ian Hodder (dir.), Contemporary Archaeology in Theory, Oxford, Blackwell Publishers, 1999 (1996), p. 615-631.

50. Silberman, Neil A., "Promised Lands and Chosen Peoples: the Politics and Poetics of Archaeological Narrative », in Nationalism, Politics and the Practice of Archaeology, ed. Philip L. Khol et Clare Fawcett, Cambridge, CUP, 1995, p. 249-250.

51. Dust Breeding, vidéo enregistrée sur DVD, $5 \mathrm{mn}$; visible sur http://greenonredgallery.com. 52. Giles, Melanie, «Bog Bodies : Representing the Dead», contribution à la conférence intitulée "Respect for Ancient British Human Remains: Philosophy and Practice», 17 novembre 2006, Manchester Museum, p. 12, retranscrite sur le site http:// www.museum.manchester.ac.uk/medialibrary/documents/respect/

bog_bodies_representing_the_dead.pdf.

\section{RÉSUMÉS}

L'attrait des corps des tourbières provient du mystère entourant leur mort et de leur capacité à bouleverser notre rapport au temps. L'ouvrage de P. V. Glob marqua S. Heaney mais aussi B. Cooke, L. le Brocquy ou H. O'Donoghue. La représentation des corps et de la violence dans les arts visuels est chez eux à la fois littérale et archétypale. Travaillant sur la fragmentation du corps, peintres ou vidéastes présentent des corps ensevelis de manière à initier une réflexion sur la blessure, l'identité et ses rapports à l'histoire nationale.

The fascination exerted by bog bodies springs from the mystery surrounding their deaths and their capacity to disrupt chronological frameworks. P. V. Glob's seminal work has had a profound 
influence on S. Heaney, as well as B. Cooke, L. le Brocquy or H. O'Donoghue. The representation of the bodies and the violence they exemplify is both literal and archetypal. The paintings and videos showing fragmented bodies explore wounds that are both organic and cultural and question the notion of identity in the face of national history.

INDEX

Mots-clés : corps des tourbières, archéologie, Le Brocquy Louis, Cooke Barrie, Rolfe Nigel, O’Donoghue Hughie, arts visuels

Keywords : archaeology, bog bodies, O'Donoghue Hughie, Cummins Pauline, visual arts

\section{AUTEUR \\ VALÉRIE MORISSON \\ Université Grenoble II}

